\title{
Synergism of FAK and tyrosine kinase inhibition in $\mathrm{Ph}^{+} \mathrm{B}-\mathrm{ALL}$
}

\author{
Michelle L. Churchman, ${ }^{1}$ Kathryn Evans, ${ }^{2}$ Jennifer Richmond, ${ }^{2}$ Alissa Robbins, ${ }^{2}$ Luke Jones, ${ }^{2}$ \\ Irina M. Shapiro, ${ }^{3}$ Jonathan A. Pachter, ${ }^{3}$ David T. Weaver, ${ }^{3}$ Peter J. Houghton, ${ }^{4}$ Malcolm A. Smith, ${ }^{5}$ \\ Richard B. Lock, ${ }^{2}$ and Charles G. Mullighan ${ }^{1}$ \\ 'Department of Pathology, St. Jude Children's Research Hospital, Memphis, Tennessee, USA. Children's Cancer Institute, \\ Lowy Cancer Research Centre, University of New South Wales, New South Wales, Sydney, Australia. ${ }^{3}$ Verastem Inc. \\ Needham, Massachusetts, USA. ${ }^{4}$ Greehey Children's Cancer Research Institute, University of Texas Health Science \\ Center San Antonio, San Antonio, Texas, USA. ${ }^{5}$ Cancer Therapy Evaluation Program, National Cancer Institute, Bethesda, \\ Maryland, USA.
}

BCR-ABL1+ $B$ progenitor acute lymphoblastic leukemia ( $\left.\mathrm{Ph}^{+} \mathrm{B}-\mathrm{ALL}\right)$ is an aggressive disease that frequently responds poorly to currently available therapies. Alterations in IKZF1, which encodes the lymphoid transcription factor lkaros, are present in over $\mathbf{8 0} \%$ of $\mathrm{Ph}^{+} \mathrm{ALL}$ and are associated with a stem cell-like phenotype, aberrant adhesion molecule expression and signaling, leukemic cell adhesion to the bone marrow stem cell niche, and poor outcome. Here, we show that FAK1 is upregulated in $\mathrm{Ph}^{+} \mathrm{B}-\mathrm{ALL}$ with further overexpression in IKZF1-altered cells and that the FAK inhibitor VS-4718 potently inhibits aberrant FAK signaling and leukemic cell adhesion, potentiating responsiveness to tyrosine kinase inhibitors, inducing cure in vivo. Thus, targeting FAK with VS-4718 is an attractive approach to overcome the deleterious effects of FAK overexpression in $\mathrm{Ph}^{+} \mathrm{B}-\mathrm{ALL}$, particularly in abrogating the adhesive phenotype induced by lkaros alterations, and warrants evaluation in clinical trials for $\mathrm{Ph}{ }^{+} \mathrm{B}-\mathrm{ALL}$, regardless of IKZF1 status.

Conflict of interest: I.M. Shapiro, J.A. Pachter, and D.T. Weaver are employed by and have equity ownership in Verastem Inc.

Submitted: December 17, 2015 Accepted: March 7, 2016 Published: April 7, 2016

Reference information: JCI Insight. 2016;1(4):e86082. doi:10.1172/ji.insight.86082.

\section{Introduction}

Acute lymphoblastic leukemia (ALL) comprises a number of subtypes defined by constellations of founding chromosomal alterations, structural alterations, and mutations that influence leukemogenesis and treatment outcome. BCR-ABL1+ $\left(\mathrm{Ph}^{+}\right)$B progenitor ALL (B-ALL) constitutes $3 \%$ to $5 \%$ of childhood and up to one-third of adult ALL cases. It is a highly aggressive disease that is often refractory to currently available therapies. Tyrosine kinase inhibitors (TKIs) that target the constitutively ABL1 kinase have revolutionized the treatment of $\mathrm{Ph}^{+}$chronic myeloid leukemia but have only partly improved the poor prognosis of $\mathrm{Ph}^{+}$ ALL. Genomic profiling studies have provided important insights into the genetic basis of these differences in outcome. In contrast to CML, which at chronic phase harbors few if any additional genomic alterations, $\mathrm{Ph}^{+}$ALL is characterized by alterations of the lymphoid transcription factor gene, $\operatorname{IKZF1}(1,2)$. In both $\mathrm{Ph}^{+}$and $\mathrm{Ph}^{-} \mathrm{ALL}, I K Z F 1$ alterations are associated with inferior treatment outcome (3). Additional genetic alterations, including deletion of the $C D K N 2 A / C D K N 2 B$ (INK4/ARF) tumor suppressor locus and deletions of $P A X 5$, are also common in $\mathrm{Ph}^{+} \mathrm{ALL}$.

In mouse models of $\mathrm{Ph}^{+} \mathrm{ALL}$, perturbations of Ikzf1, including loss-of-function deletions and expression of the dominant-negative isoform IK6 (deletion of exons 4-7), result in the acquisition of stem cell-like features, enhanced self-renewal, overexpression of adhesion molecules, and upregulation of focal adhesion kinase (FAK), in part due to the derepression of stem cell and adhesion genes normally silenced by IKZF1 $(4,5)$. This results in leukemic cell adhesion to other tumor cells as well as stromal cells of the bone marrow microenvironment, aberrant localization of leukemic cells to the periarteriolar bone marrow niche, and reduced sensitivity to therapy (4). A detailed understanding of the interactions between leukemic cells and the bone marrow microenvironment is important to fully understand leukemogenesis, tumor maintenance, and resistance to therapy. These recent insights indicate that aberrant interactions of leukemic cells with the microenvironment are dictated by genetic alterations in leukemic cells and that targeting pathways that influence the tumor cell microenvironment interactions may prove indispensable in improving responses to standard therapies and reducing the incidence of relapse for high-risk, IKZF1-mutated $\mathrm{Ph}^{+} \mathrm{B}-\mathrm{ALL}$. 
FAK is a cytoplasmic, nonreceptor protein tyrosine kinase that plays a central role in integrin-mediated signaling, whereby integrin binding to extracellular matrix (ECM) proteins stimulates tyrosine phosphorylation of FAK at residue Y397 (6). Adhesion of cells to ECM proteins increases phosphorylation and activation of FAK (7), which initiates signaling cascades involving Src, p130Cas, paxillin, STAT3, and ERK that regulate cellular processes, such as adhesion, proliferation, survival, and cell migration (8-10). These processes are deregulated by overexpression or aberrant activation of FAK in several cancers, promoting tumor growth and metastasis (11). Targeted inhibition of the FAK pathway is a promising therapeutic approach to broadly suppress various tumor-promoting signaling pathways that converge on FAK activation.

VS-4718 is a potent, selective, and orally bioavailable FAK inhibitor currently under evaluation in subjects with various solid tumors in a phase I clinical trial (ClinicalTrials.gov NCT01849744); however, efficacy in hematological malignancies had not been evaluated. We previously showed efficacy of nonclinical, tool compound FAK inhibitors in vitro, suggesting that these agents may represent a new therapeutic option to overcome TKI resistance in vivo (4). We show here that FAK inhibition with VS-4718 attenuated the survival, clonogenicity, and adhesion of $\mathrm{Ph}^{+}$B-ALL leukemic cells, both in IKZF1 wild-type and mutant leukemic cells. Ultimately, VS-4718 synergized with dasatinib to improve outcome of engineered and xenografted mouse models of $\mathrm{Ph}^{+} \mathrm{ALL}$.

\section{Results}

FAK pathway upregulation in $P h^{+} B-A L L$, with further activation in Ikzf1-mutated $P h^{+} B-A L L$ cells. Gene expression profiling of primary mouse $A r f^{\prime-}$ pre-B cells expressing empty vector, BCR-ABL1, or EBF1-PDGFRB revealed that Fak1 is overexpressed in $\mathrm{Ph}^{+}$cells (Figure 1A). Expression of EBF1-PDGFRB, a fusion identified in Ph-like B-ALL that results in constitutive kinase activity, cytokine-independent cell line proliferation, and leukemogenesis (12), did not result in upregulation of Fak1 (Figure 1A). The expression of the dominant-negative Ikaros isoform, IK6, in combination with BCR-ABL1 resulted in further upregulation of Fak1, whereas IK6 did not increase Fak1 expression in empty vector or EBF1-PDGFRB-expressing pre-B cells (Figure 1A). Interestingly, IK6 expression upregulated Fak2 (also known as Ptk2B or Pyk2) in non- $\mathrm{Ph}^{+}$ cells but not in BCR-ABL1-expressing cells (Figure 1, A and B). RNA (mRNA) and protein sequencing confirmed that FAK1 was overexpressed in IK6-expressing $\mathrm{Ph}^{+}$pre-B cells, and FAK2 expression was relatively high in all BCR-ABL1-expressing cells (Figure 1, B and C). Although a broad range of FAK1 expression was observed by mRNA sequencing of $19 \mathrm{Ph}^{+}$and $109 \mathrm{Ph}$-like patient samples regardless of IKZF1 status (Figure 1D), activation of the pathway was revealed by reverse-phase protein array in human $\mathrm{Ph}^{+} \mathrm{IK}^{+} \mathrm{B}-\mathrm{ALL}$ cells compared with $\mathrm{Ph}^{+} \mathrm{B}-\mathrm{ALL}$ cells with intact IKZF1(Figure 1E).

FAK inhibition decreases survival, clonogenicity, and adhesion of Ikzf1-mutated $P h^{+} B$-ALL cells. As a single agent, VS-4718 is cytotoxic to primary human $\mathrm{Ph}^{+} \mathrm{IK} 6$-expressing B-ALL cells and mouse $A r f^{\prime-}$ or $I k z f 1^{+/}$ pre-B cells expressing BCR-ABL1 with and without IK6 or a dominant-negative IKZF1 point mutation, D186A, at concentrations above $0.1 \mu \mathrm{M}$ (Figure 2A). Ikzf1 haploinsufficiency and IK6 expression resulted in less sensitivity to FAK inhibition compared with cells with intact $I k z f 1$, whereas cells harboring IKZF1 D186A were as sensitive as cells expressing empty GFP vector (MSCV-IRES-GFP [MIG]; Figure 2A). Human $\mathrm{Ph}^{+}$cells underwent apoptosis in response to exposure to VS-4718 ex vivo (Figure 2B).

Ikzf1 alterations confer aberrant hematopoietic stem cell-like properties to $\mathrm{Ph}^{+}$pre-B cells, including increased self-renewal and adhesion to ECMs in vitro and stromal cells in the bone marrow niche (4). VS-4718 attenuated the capability of cells with or without $I k z f 1$ alterations to form colonies from single cells at concentrations that do not affect cell viability (Figure 2C). FAK inhibition also blocked adhesion to fibronectin fragment monolayers regardless of Ikzf1 status; although the greatest effects were observed in cells harboring Ikzf1 alterations (Figure 2D). VS-4718 treatment inhibited FAK pathway signaling, as revealed by reverse-phase protein array analysis of key targets in human $\mathrm{Ph}^{+} \mathrm{IK} 6^{+} \mathrm{B}$-ALL cells (Figure $2 \mathrm{E}$ ).

In the bone marrow niche, $A r f^{\prime-} \mathrm{Ph}^{+} \mathrm{IK}^{+}$leukemic cells adopt a spindle-like adherent phenotype, in stark contrast to the typical spherical shape of pre-B cells (4). To visualize the effects of VS-4718 on adherence of cells in the bone marrow niche, we transplanted GFP-labeled $\mathrm{Arf}^{-} \mathrm{Ph}^{+} \mathrm{IK}^{+}$cells into recipient mice that were randomized and treated daily with vehicle or VS-4718 for 3 days, starting 24 hours after transplant. Calvaria were harvested and immediately imaged by multiphoton microscopy. Vehicle-treated mice displayed the adherent, irregularly shaped spindle-like morphology, whereas VS-4718 treatment abrogated the adhesive phenotype of the cells, without directly affecting engraftment or viability of cells (Figure 
A

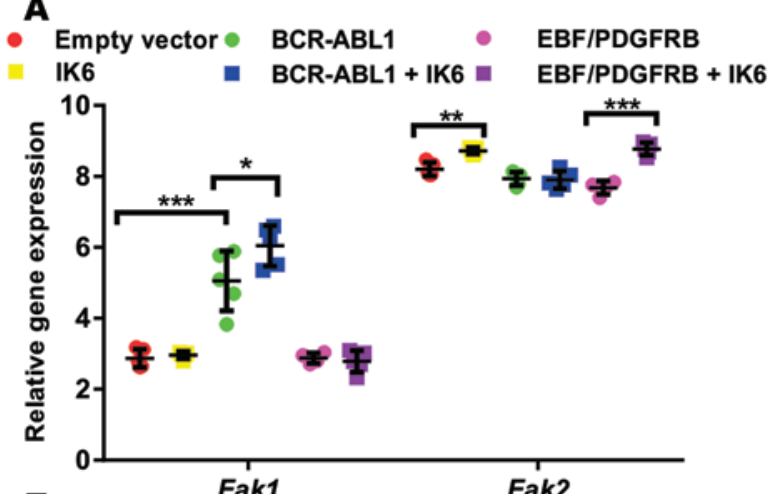

E

Fak1

들
BCR-ABL1

- BCR-ABL1 + IK6

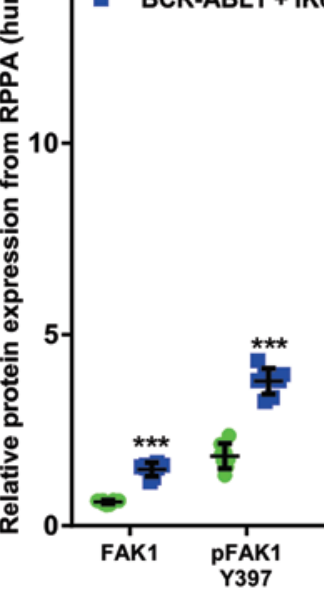

B

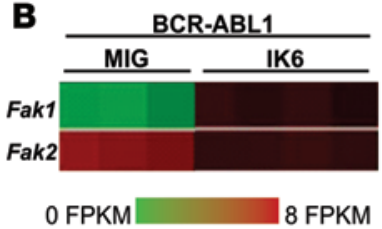

C

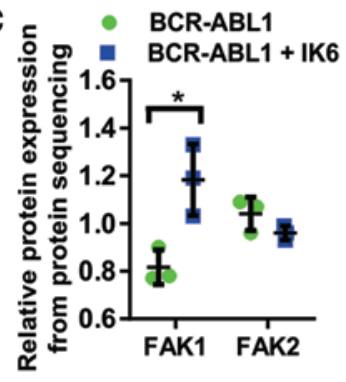

D

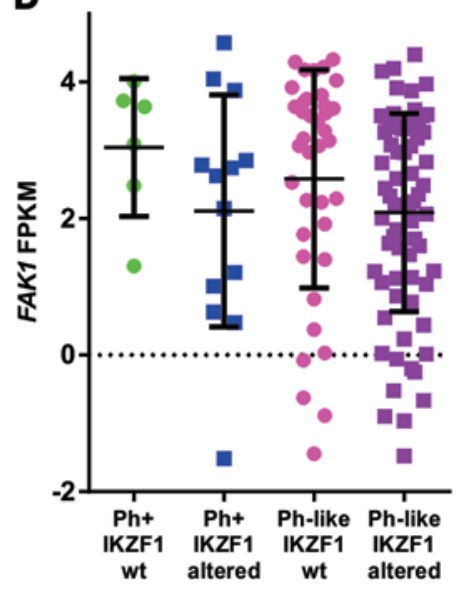

Figure 1. Focal adhesion kinase pathway upregulation in BCR-ABL1 B progenitor acute lymphoblastic leukemia, with further upregulation by IKZF1 alteration. (A) Gene expression profiling, (B) mRNA sequencing, and (C) protein sequencing of focal adhesion kinase 1 (Fak1) and Fak2 in murine Arf ${ }^{-1-}$ pre-B cells. (D) mRNA sequencing of human BCR-ABL1+ $\left(\mathrm{Ph}^{+}\right)$and Ph-like patient cohorts. (E) Reverse-phase protein array (RPPA) analysis of FAK1 expression and downstream targets of FAK signaling in human $\mathrm{Ph}^{+} \mathrm{B}$ progenitor acute lymphoblastic leukemia (B-ALL) (h9407) cells with and without IKZF1 alteration (IK6). Data represent averages \pm SD; $n=5$ technical replicates each in A, 3 (MIG) and 4 (IK6) biological replicates in B, 3 biological replicates in C, $19 \mathrm{Ph}^{+}$and $109 \mathrm{Ph}$-like patients in $\mathbf{D}$, and 4 technical replicates of 2 biological replicates in $\mathbf{D}$. ${ }^{*} P \leq 0.05,{ }^{*} P \leq 0.005,{ }^{* *} P \leq 0.0005,2$-tailed Student's $t$ test. MIG, MSCV-IRES-GFP (empty GFP vector); FPKM, fragments per kilobase of transcripts per million mapped reads.

3, A and B). Quantification of the spindle-like or typical round morphology of cells revealed a reduction in the number of cells with an adherent, irregular appearance in the bone marrow niche after VS-4718 treatment (Figure 3C).

VS-4718 synergizes with dasatinib in affecting cell survival, adhesion, and inhibition of downstream targets of FAK. Dasatinib is a TKI that targets BCR-ABL1; it is widely used in frontline treatment of $\mathrm{Ph}^{+} \mathrm{B}$-ALL. To assess the combinatorial effects of dasatinib and FAK inhibition, we performed cell viability assays using $A r f^{\prime-} \mathrm{Ph}^{+}$pre-B cells expressing either empty vector (MIG) or IK6 at fixed drug ratios to determine combination index (CI) values. Dasatinib synergized with VS-4718 in decreasing the survival of cells at increasing concentrations of drug (Figure 4A). CI values ranged from 0.68 to 0.91 for cells expressing empty vector (MIG), suggesting synergistic to additive effects, whereas IK6-expressing cells displayed CI values indicative of strong synergistic effects of combination therapy (Figure 4B). Four primary human $\mathrm{Ph}^{+}$ B-ALL lines harboring IKZF1 alterations (ALL-4 [IK6], ALL-55 [deletion of exons 2-7], ALL-56 [deletion of exons 2-7], and h9407 [IK6] cells) displayed highly variable responses to dasatinib and VS-4718 as single agents ex vivo; however, exposure to both drugs decreased the $\mathrm{IC}_{50}$ values compared with values after exposure to either alone for all lines except ALL-55, which was refractory to both drugs (Figure 4, C and D).

Given that VS-4718 and dasatinib synergize in decreasing cell viability, we explored their effects on cell adhesion by exposing $A f^{\prime-} \mathrm{Ph}^{+}$pre-B cells expressing either empty vector (MIG) or IK6 to RetroNectin 
A

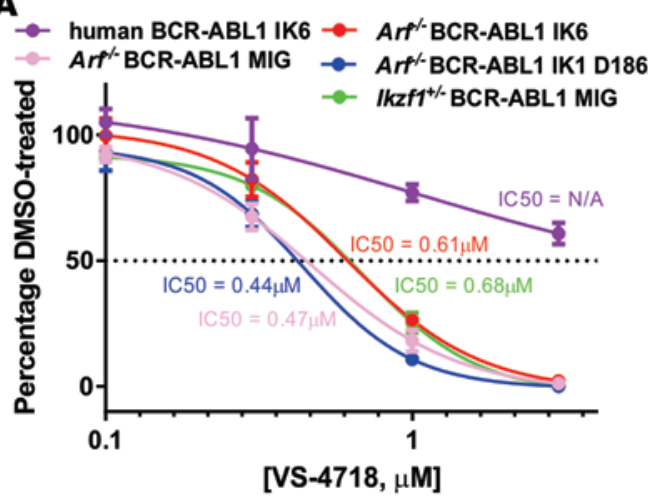

B

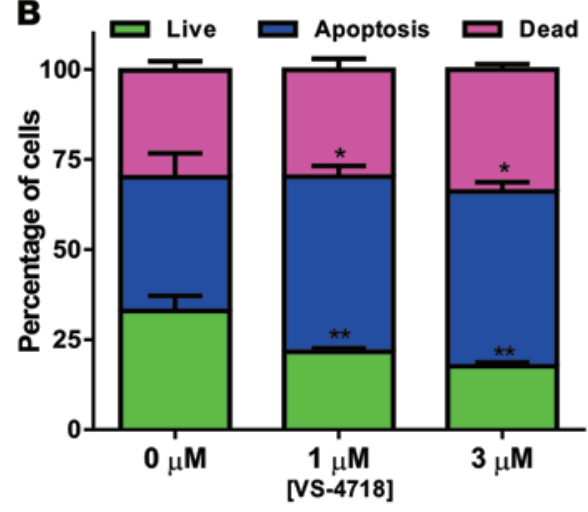

D
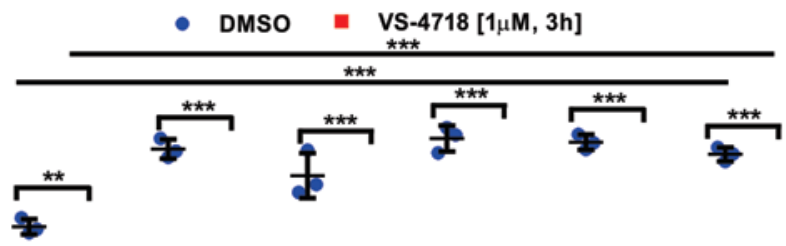

$\frac{2}{2}$

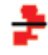

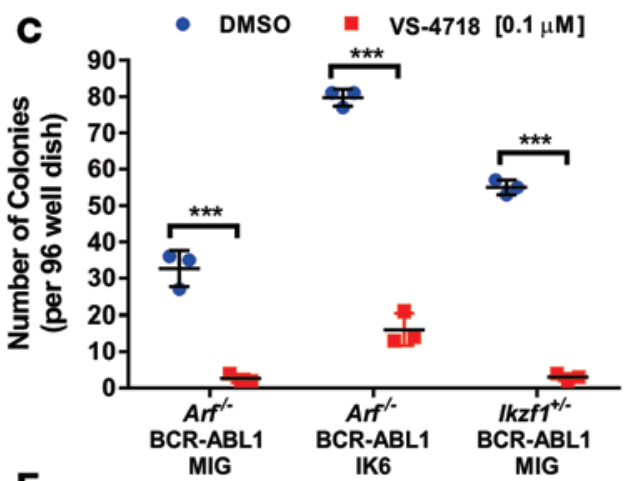

E

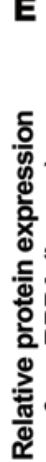
MIG IK6
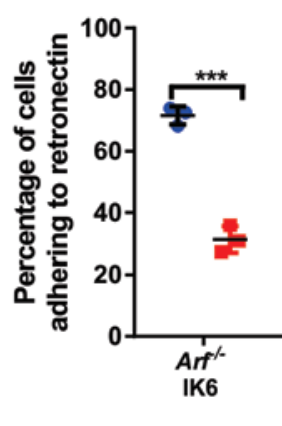

.

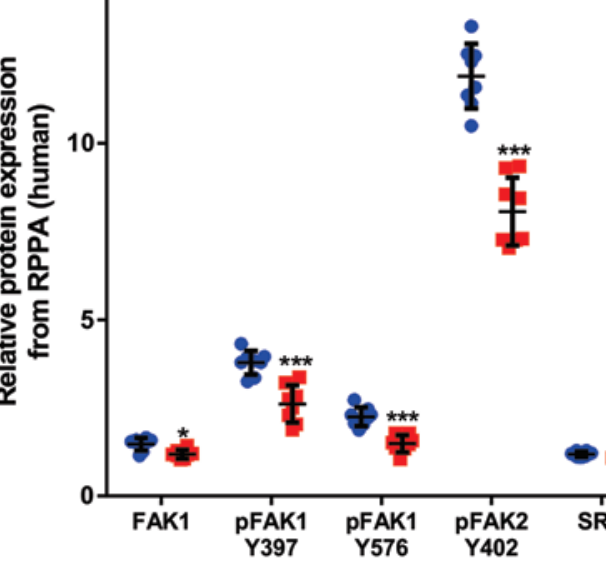

- Vehicle $=$ VS-4718 $(0.1 \mu \mathrm{M})$
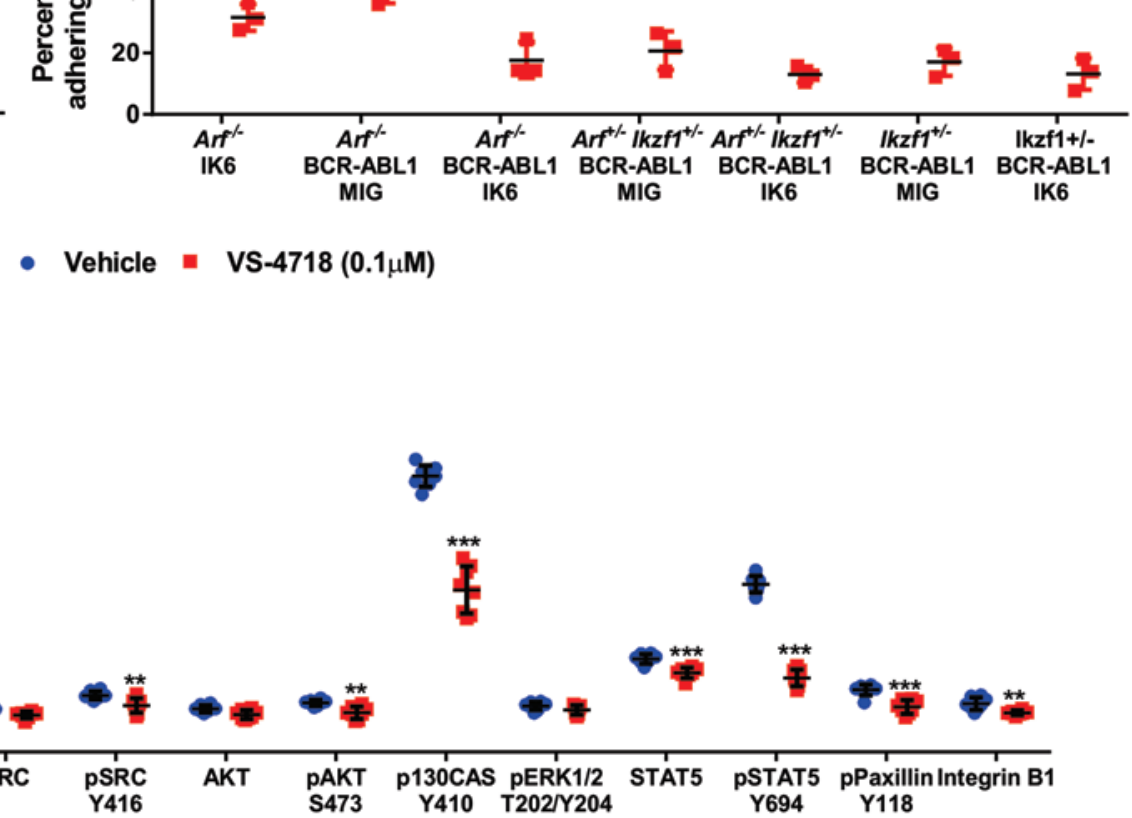

Figure 2. Focal adhesion kinase inhibition decreases survival, clonogenicity, and adhesion of BCR-ABL1+ $B$ progenitor acute lymphoblastic leukemia cells in vitro. (A) Human BCR-ABL1+ $\left(\mathrm{Ph}^{+}\right) \mathrm{IK} 6^{+} \mathrm{h} 9407 \mathrm{~B}$ progenitor acute lymphoblastic leukemia (B-ALL) cells and murine $\mathrm{Ph}^{+}$pre-B cells expressing empty vector (MIC), IKG ${ }^{+}$, or the IK1 D186A point mutation have decreased viability when treated with VS-4718 at concentrations above $0.1 \mu \mathrm{M}$. (B) Decreased numbers of VS-4718-treated h9407 cells are the result of increased apoptosis. (C) The colony-forming potential of single cell-sorted $\mathrm{Ph}^{+}$murine pre-B cells of the indicated genotypes is greatly reduced in the presence of VS-4718 at a concentration ( $0.1 \mu \mathrm{M})$ that does not affect cell proliferation or viability. (D) Murine pre-B cells of the indicated genotypes are less adherent to RetroNectin monolayers due to focal adhesion kinase (FAK) inhibition after short-term

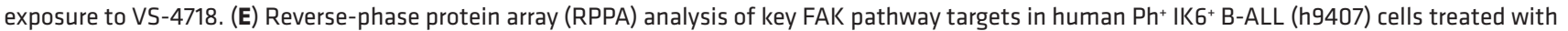
either vehicle or VS-4718 for 24 hours in vitro. Data represent averages \pm SD; $n=3$ biological replicates each in $\mathbf{A}$ and $\mathbf{C}, 3$ technical replicates in $\mathbf{B}$ and $\mathbf{D}$, and 4 technical replicates of 2 biologic replicates in $\mathbf{E} .{ }^{*} P \leq 0.05,{ }^{* *} P \leq 0.005,{ }^{* * *} P \leq 0.0005,2$-tailed Student's $t$ test in B-E with 2-way ANOVA in $\mathbf{E}$. MIG, MSCV-IRES-GFP (empty GFP vector).

monolayers after single and combination treatments. As expected, IK6-expressing cells were more adherent after a short (30-minute) exposure to RetroNectin than their empty vector-expressing counterparts (ref. 4 and Figure 4E). Alone, FAK inhibition with VS-4718 or ABL inhibition with dasatinib significantly reduced the ability of IK6-expressing cells to adhere to RetroNectin, whereas the number of adherent MIG-expressing cells was slightly, yet not significantly reduced after VS-4718 or dasatinib treatment alone 

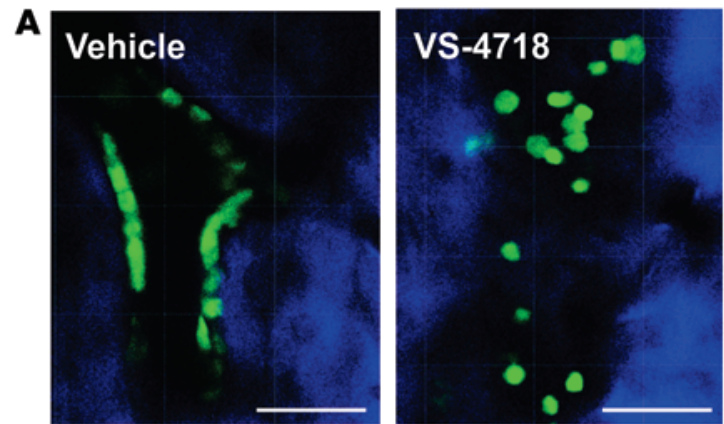

B

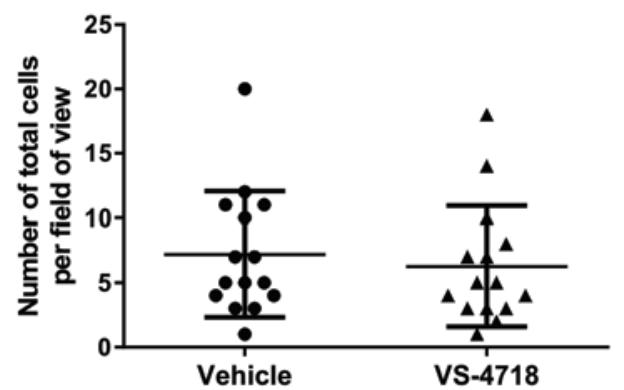

C

$\square$ Adherent, spindle-like

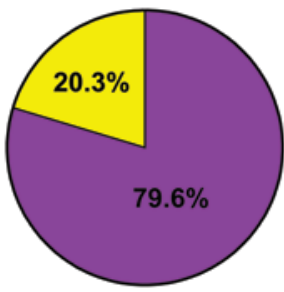

Vehicle

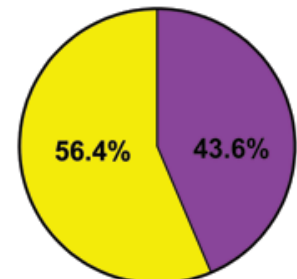

VS-4718
Figure 3. VS-4718 inhibits cell-to-stroma adhesion of leukemic murine Arf $^{-/}$BCR-ABL1 pre-B cells in the bone marrow niche. (A) Calvarial imaging of $\mathrm{Arf}^{-1-} \mathrm{BCR}-\mathrm{ABL} 1(\mathrm{Ph}) \mathrm{IK}^{+}-\mathrm{CFP}$ leukemic cells in the bone marrow niche, showing stromal adhesion of $\mathrm{GFP}^{+}$cells in vehicle-treated mice and the nonadhesive "round" phenotype of cells in the bone marrow niche of VS-4718-treated mice. (B) The total number of cells observed per field of view in each calvarium was enumerated to determine whether VS-4718 treatment effects leukemic cell viability or engraftment at this dosage $(50 \mathrm{mg} / \mathrm{kg}) \cdot n=5$ fields of view each for 3 calvaria. (C) The phenotype of leukemic cells was categorized by the appearance of "spindle-like" adherent cells or "round" nonadherent appearing cells, indicating loss of adhesion to surrounding stroma due to FAK inhibition with VS-4718. $n=$ 108 cells from vehicle-treated mice and 94 cells from VS-4718-treated mice ( 3 mice per group, 5 fields of view each). Scale bar: $40 \mu \mathrm{M}$.

(Figure 4E). In contrast, the ability of both MIG- and IK6-expressing cells to adhere to RetroNectin was significantly attenuated following combination dasatinib and VS-4718 treatment, and the reduction was greater than that with either drug alone (Figure 4E). Enhanced inhibition of p-P130Cas, a downstream target of FAK, was observed in the adherent fraction of cells, chosen due to maximal activation of the pathway by ECM exposure at 15 minutes, 30 minutes, and 2 hours after treatment with a combination of VS-4718 and dasatinib on RetroNectin (Figure 4F).

Combination treatment with VS-4718 and dasatinib improves therapeutic outcome of $\mathrm{Ph}^{+} B-A L L$. For in vivo efficacy studies, BCR-ABL1-transduced pre-B cells expressing luciferase were derived from $\mathrm{Arf}^{\prime-}$ donor mice and subsequently transduced with either empty GFP vector (MIG) or IK6-GFP, sorted, and injected into sublethally irradiated wild-type recipients. Mice were monitored for leukemic burden by detecting luciferase activity in vivo by live animal bioluminescence imaging (Figure $5 \mathrm{~A}$ ). By day 7, mice had measurable tumor burden and treatment was commenced. Untreated animals from both empty vector and IK6 groups succumbed to disease by day 14 (4), and VS-4718 treatment alone did not affect leukemic burden or survival (data not shown). Although inefficacious alone, VS-4718 in combination with dasatinib dramatically decreased leukemic burden and extended the lives of both MIG and IK6 cohorts, with one long-term survivor from the IK6 group that underwent a complete remission, despite the cessation of treatment (Figure $5, \mathrm{~B}$ and $\mathrm{C})$. At the time of sacrifice for moribund animals that succumbed to disease, spleen weights were slightly reduced in combination-treated mice (Figure 5D).

To further test the efficacy of VS-4718 in vivo, human $\mathrm{Ph}^{+} \mathrm{IK}^{+}$(h9407) cells were xenografted into NOD/SCID $\gamma$-null (NOD.Cg-Prkdcsid $I l 2 \mathrm{rg}^{\mathrm{tm} l \mathrm{Wjl}} / \mathrm{SzJ}$ [NSG]) mice, harvested, marked with luciferase ex vivo, retransplanted into mice for expansion, and then harvested, sorted, and retransplanted for in vivo efficacy studies tracing leukemic burden by monitoring luciferase activity. Preliminary studies revealed that VS-4718 was not effective as a single agent (Figure 6A); however, in combination with dasatinib, FAK 


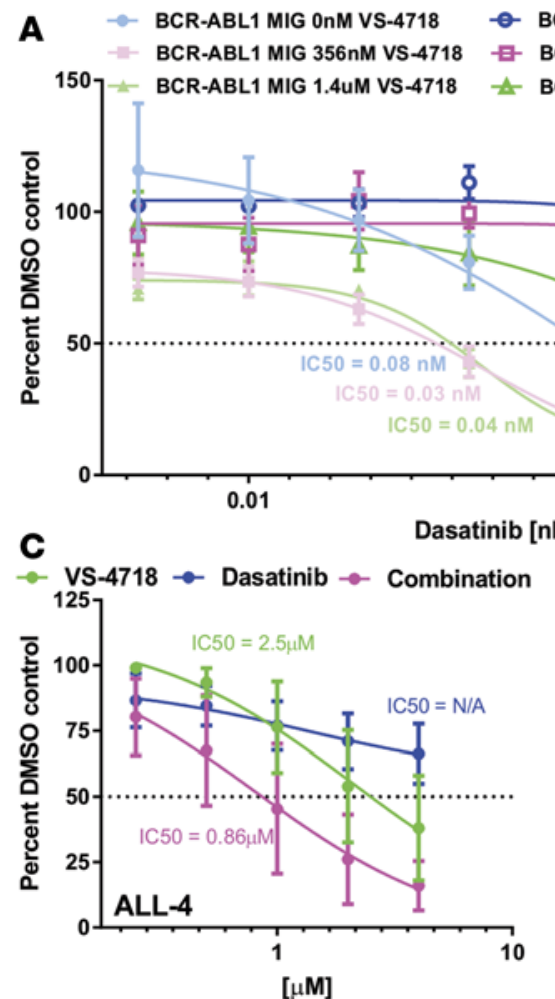

BCR-ABL1 IK6 OnM VS-4718

BCR-ABL1 IK6 356nM VS-4718

BCR-ABL1 IK6 1.4uM VS-4718

IC $50=0.25 \mathrm{nM}$
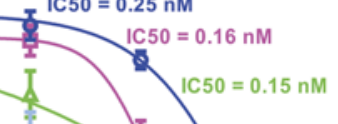
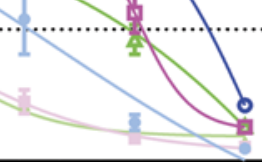

0.1

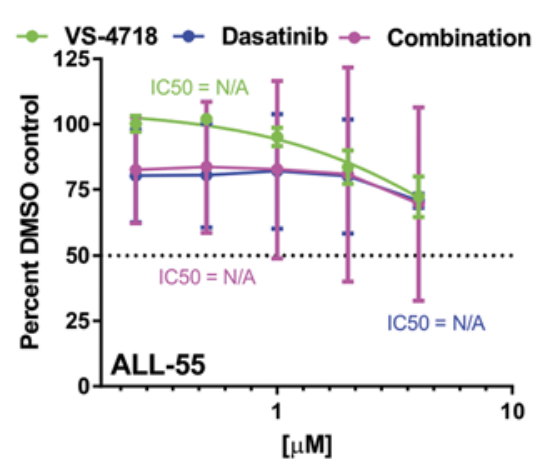

B

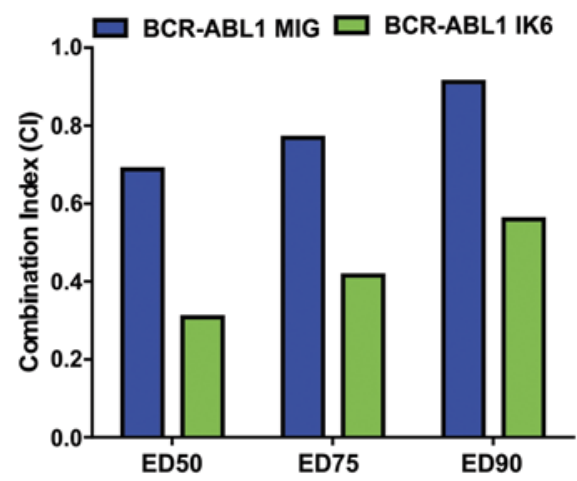

D_ $0 \mu \mathrm{M}$ VS-4718 $-1 \mu \mathrm{M}$ VS-4718

E DMSO $\triangle$ Dasatinib

$-0.3 \mu \mathrm{M}$ VS $-4718-3 \mu \mathrm{M}$ VS -4718

= VS-4718 - Dasatinib + VS-4718
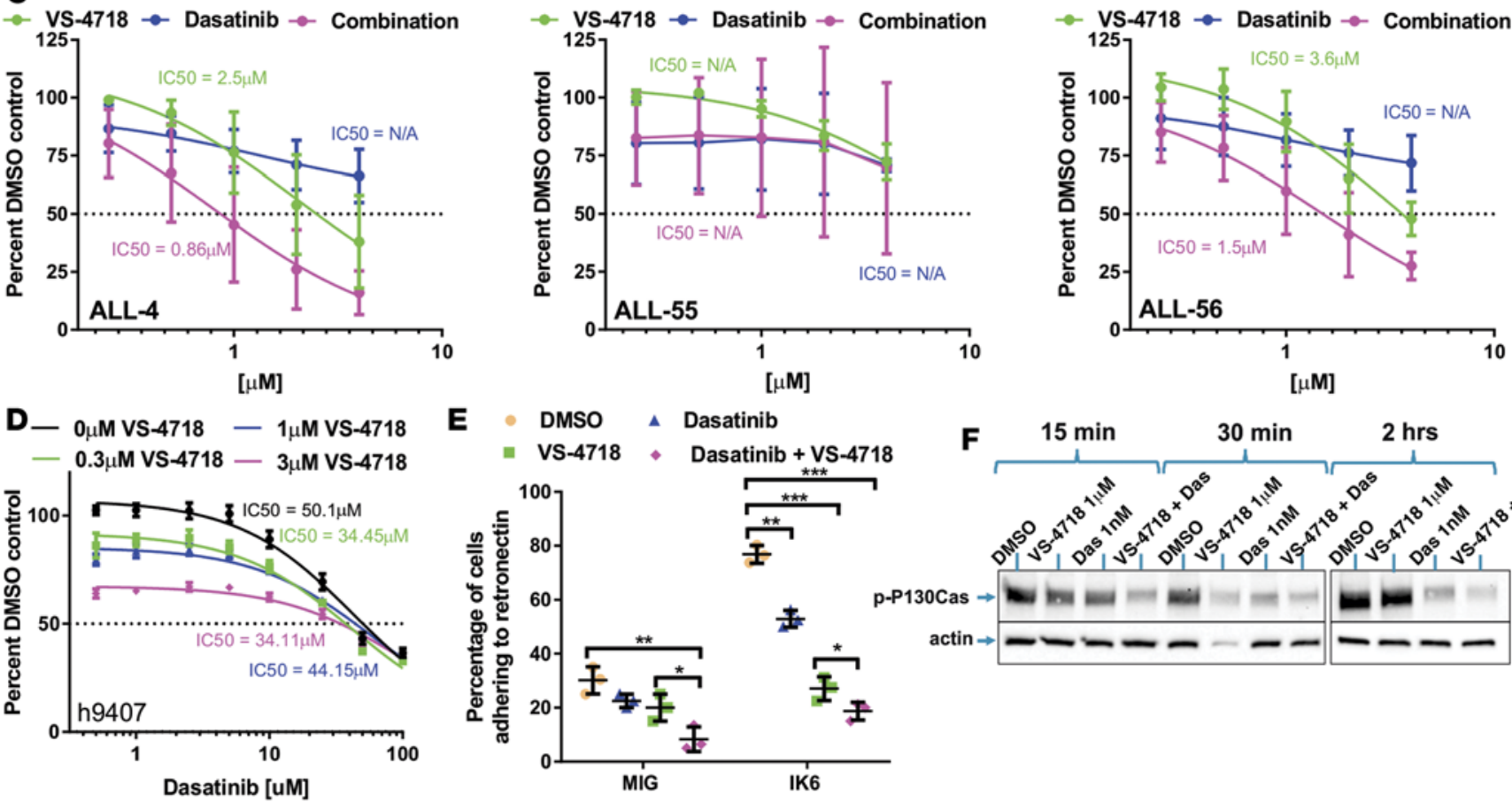

$[\mu \mathrm{M}]$

Figure 4. VS-4718 synergizes with dasatinib to decrease cell survival and adhesion of BCR-ABL1 B progenitor acute lymphoblastic leukemia cells. (A) VS-4718 synergizes with dasatinib to lower the IC ${ }_{50}$ of Arf $^{-1-}$ BCR-ABL1 (Ph) pre-B cells expressing MIC or IK6 in cell viability assays. (B) Combination index (CI) values from viability assays shown in A indicate synergistic to additive effects of VS-4718 and dasatinib on MIC cells and strong synergy for IK6 cells ( $\mathrm{Cl} \leq 0.3$ = strong synergy; $0.3<\mathrm{Cl} \leq 0.85=$ synergy; $0.85<\mathrm{Cl} \leq 1.2$ = additive; $1.2<\mathrm{Cl} \leq 3.3=$ antagonism; $\mathrm{Cl}>3.3=$ strong antagonism). (C) Cell viability assays with human ALL-4 and ALL-56 cells displayed synergism between VS-4718 and dasatinib; however, the survival of ALL-55 cells was not decreased with combination treatment. (D) Human h9407 B progenitor acute lymphoblastic leukemia cells are less sensitive to increasing concentrations of dasatinib alone compared with combination treatment with VS-4718 in cell viability assays ex vivo. (E) Adhesion assays were used to detect the effects of VS-4718 and dasatinib on the ability of $\mathrm{Arf}^{-/-}$Ph pre-B cells expressing MIG or IK6 to adhere to RetroNectin monolayers in vitro. Combination treatment with VS-4718 and dasatinib synergistically abolished cellular adhesion to RetroNectin. (F) Downstream FAK signaling is inhibited synergistically by VS-4718 and dasatinib in the adherent fraction of cells from the RetroNectin assays shown in $\mathbf{E}$, as detected by Western blotting for $\mathrm{p}$-P130Cas expression at 15 minutes, 30 minutes, and 2 hours of exposure to drug. Data represent averages \pm SD of 3 technical replicates for each group in $\mathbf{A}-\mathbf{E} ;{ }^{*} P \leq 0.05,{ }^{* *} P \leq 0.005$, ${ }^{* * *} P \leq 0.0005$, 2-tailed Student's $t$ test. MIG, MSCV-IRES-GFP (empty GFP vector); Das, dasatinib.

inhibition significantly reduced leukemic burden (Figure 6B). Survival was not a reliable readout for drug efficacy using h9407 cells, as mice were able to harbor high leukemic burden without manifesting symptoms of illness; hence, luciferase quantifications were employed as a measure of efficacy, and animals were sacrificed at the end of the study. Activity against a second human $\mathrm{Ph}^{+} \mathrm{IK} 6^{+} \mathrm{B}-\mathrm{ALL}$ line, ALL-4, was evaluated by monitoring human CD45 (hCD45) in peripheral blood, and survival was commensurate with disease burden. Again, VS-4718 provided no benefit as a single agent; however, it displayed remarkable efficacy in combination with dasatinib, whereby disease progression was markedly slowed and survival was prolonged on combination therapy (Figure 6, C and D). The number of circulating and 

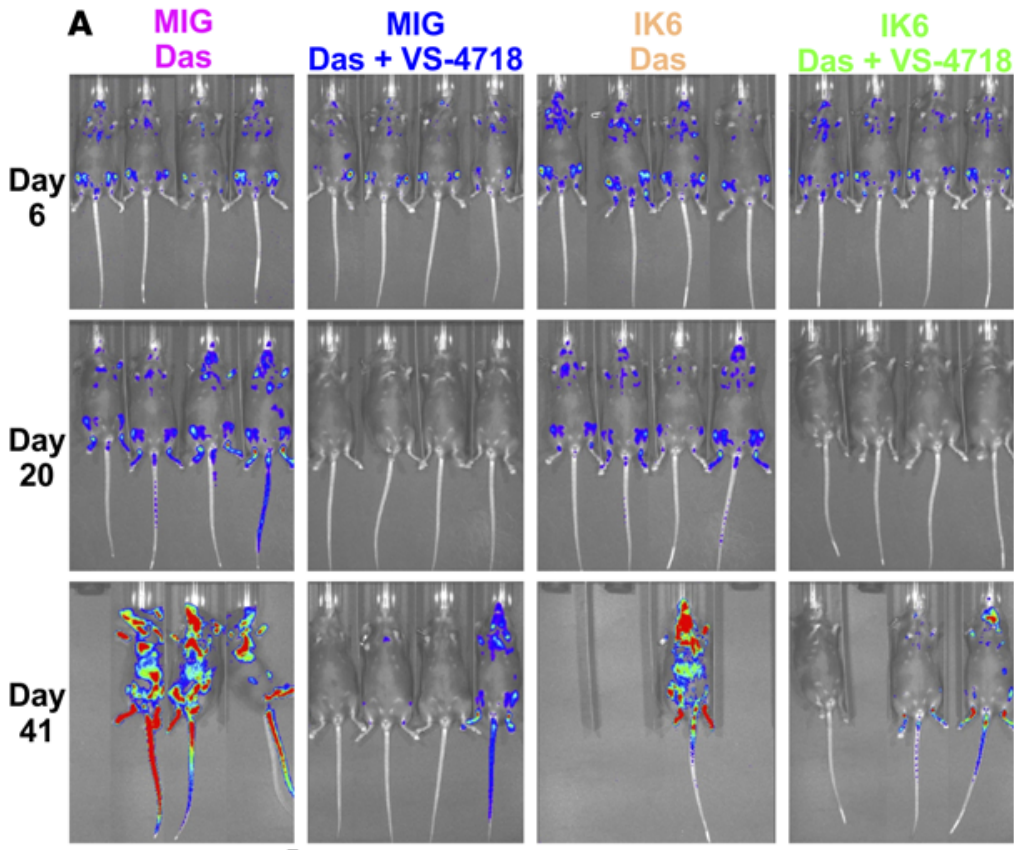

Luminescence $\left(\times 10^{7}\right)$

B - BCR-ABL1 MIG Das

$$
0.5
$$

- BCR-ABL1 MIG Das + VS-4718

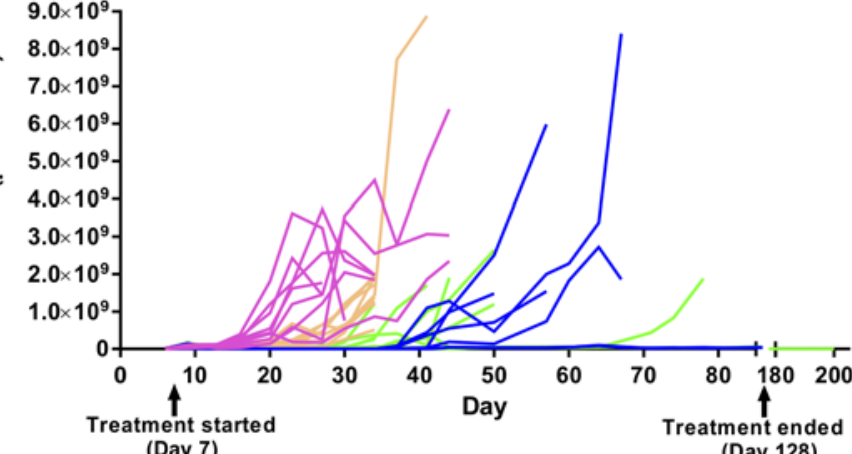

(Day 7)

C

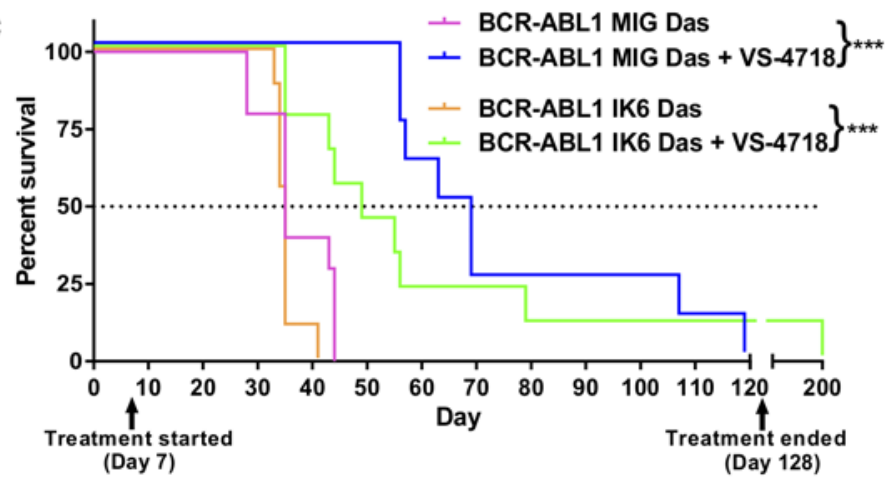

D

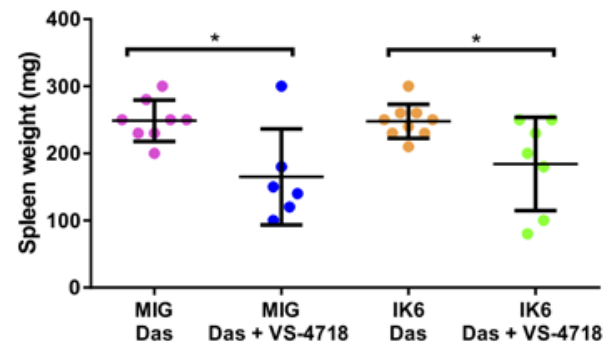

Figure 5. Combination VS-4718 and dasatinib therapy decreases leukemic burden and increases survival of murine BCR-ABL1 B progenitor acute lymphoblastic leukemia. (A) Live whole-animal imaging of representative mice transplanted with Arf $^{-1-}$ BCR-ABL1 (Ph) pre-B cells expressing luciferase, with and without IK6, used for testing the in vivo efficacy of VS-4718 and dasatinib against murine $\mathrm{Ph}^{+}$ B progenitor acute lymphoblastic leukemia. (B) Quantification of luciferase activity to monitor the progression of disease during treatment. (C) Kaplan-Meier survival curve displaying increased survival on combination VS-4718 and dasatinib therapy, with one long-term remission achieved until the surviving mouse was sacrificed with no indication of leukemia at day 200. (D) Combination treatment slightly reduced spleen weight, as observed at the time of sacrifice when mice were moribund. $n=10$ mice per group; ${ }^{*} P \leq 0.05$ Student's $t$ test, ${ }^{* *} P \leq 0.0005$, Mantel-Cox test. MIG, MSCV-IRES-GFP (empty GFP vector); Das, dasatinib 
organ-infiltrating hCD $45^{+}$blasts was reduced to nearly undetectable levels at day 28 after treatment with dasatinib and VS-4718 (Figure 6, C and E). Although a durable remission was achieved on combination therapy, mice relapsed between 1 and 3 weeks after completion of treatment on day 28 and ultimately succumbed to florid disease (Figure 6, C-E). Analyses of spleen samples by Western blot and ELISA confirmed that phosphorylation of FAK at residue Y397 (pFAK-Y397), which is indicative of FAK activation, was inhibited by in vivo treatment with VS-4718 (Figure 6, F and G).

\section{Discussion}

Here, we have shown that FAK inhibition attenuates the aberrant adhesive and stem cell-like phenotypes induced by IKZF1 alterations in $\mathrm{Ph}^{+} \mathrm{ALL}$ and show that combined therapy with FAK inhibitors and the $\mathrm{ABL}$ inhibitor dasatinib is remarkably effective in abrogating leukemic cell growth in vivo. Indeed, this combinatorial approach induced durable remission without recurrence following cessation of therapy.

Genetic alterations resulting in loss of function or dominant-negative inhibition of B lineage transcription factors are a hallmark of B progenitor ALL (13), with significant differences observed in the nature of alteration between B-ALL subtypes. Notably, favorable risk subtypes such as ETV6-RUNX1 ALL harbor deletions of $P A X 5$, which is required for $\mathrm{B}$ lineage commitment and maintenance, in one-third of cases but rarely have alterations of $I K Z F 1$. In contrast, $\mathrm{Ph}^{+} \mathrm{ALL}, \mathrm{Ph}$-like ALL, and ERG-dysregulated ALL have a high frequency of $I K Z F 1$ alterations $(1,2)$. In contrast to $I K Z F 1, P A X 5$ alterations, which are associated with poor prognosis in $\mathrm{Ph}^{+}$and $\mathrm{Ph}$-like $\mathrm{ALL}$, are not consistently associated with poor outcome, suggesting distinct roles of each genetic alteration in leukemogenesis and responsiveness to therapy.

Multiple studies have shown that loss of function of $P A X 5, E B F 1$, and $I K Z F 1$ increases the penetrance of mouse B-ALL either in Ph-driven spontaneous or mutagenesis-primed models (14-16). In $\mathrm{Ph}^{+} \mathrm{ALL}$, loss of IKZF1 activity shifts the phenotype from a myeloid, CML-like disease to an aggressive, transplantable lymphoid leukemia, without expanding the pool of lymphoid precursors (4). Alterations of IKZF1, including loss-of-function deletions, dominant-negative alleles, and dominant-negative point mutations, also result in resistance to TKI-based therapy independent of inhibition of the ABL1 kinase (4).

The mechanistic basis of $I K Z F 1$-mediated resistance to therapy has now been elucidated. IKZF1 is a zinc finger transcription factor that mediates transcriptional activation and repression, in part through interactions and recruitment of histone-modifying complexes, such as the nucleosome remodeling and deacetylase complex $(17,18)$, transcriptional elongation complex positive-transcription elongation factor $b(19)$, and polycomb repressor complex 2 (20). The dominant-negative deletions and mutations of IKZF1 remove or perturb function of the N-terminal zinc fingers, resulting in loss of DNA-binding activity but retention of dimerization, resulting in cellular mislocalization $(4,21)$. The resulting loss or inhibition of activity dysregulates and activates multiple cellular pathways, including reactivation of hematopoietic stem cell and adhesion pathways (4). Key adhesion molecules expressed include CD90 (THY-1) and integrins, particularly ITGA5, which, upon engagement of ligand, stimulate intracellular signaling pathways $(4,5)$. FAK expression is elevated in IKZF1-mutated leukemias, and phosphorylation of FAK itself, as well as downstream effectors, is likely augmented by the direct action of activated ABL1 itself.

We previously showed that this phenotype was accompanied by aberrant self-renewal and markedly enhanced intracellular and cell-stromal adhesion, resulting in extravasation and relocalization of leukemic cells to a periarteriolar location in the bone marrow niche (4), which in part may account for the aggressive phenotype of IKZF1-mutated ALL observed in mouse models characterized by extramedullary invasion and aggressive central nervous system infiltration. Using high-content screening, we showed that retinoids, specifically rexinoid $\mathrm{X}$ receptor agonists, potently reversed this phenotype, abrogating self-renewal and inducing differentiation. In part, retinoids exerted this effect by inducing expression of wild-type IKZF1, relocalizing the protein to the nucleus, suppressing expression of stem cell and adhesion molecules, and abrogating adhesion. This also suggested that approaches directly targeting these adhesion pathways may also be efficacious.

Our results confirm this, showing that FAK inhibition blocks phosphorylation of FAK and downstream effectors. With the exception of FLT3, VS-4718 is specific for FAK at low concentrations in vitro $(0.1 \mu \mathrm{M})$, with partial inhibition of additional kinases at higher concentrations, including ACK1 (activated Cdc42-associated tyrosine kinase 1), aurora-A, CDK2 (cyclin-dependent kinase 2), insulin receptor, LCK (lymphocyte-specific protein tyrosine kinase), and TRKA (tropomyosin-related kinase A) (22). Importantly, little single-agent activity was observed, whereas the combination of dasatinib and FAK inhibition 


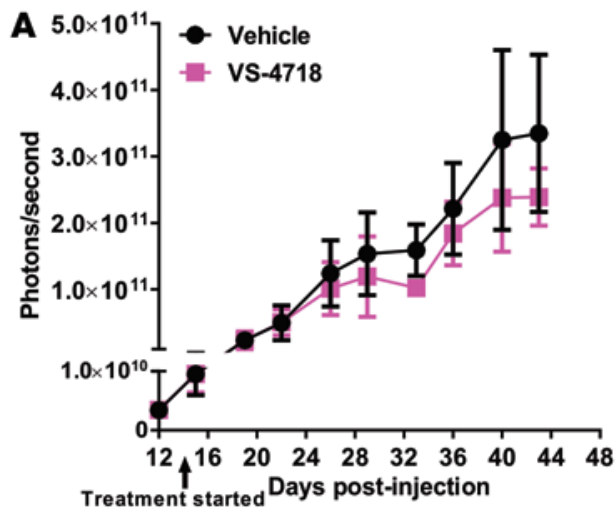

(Day 14)
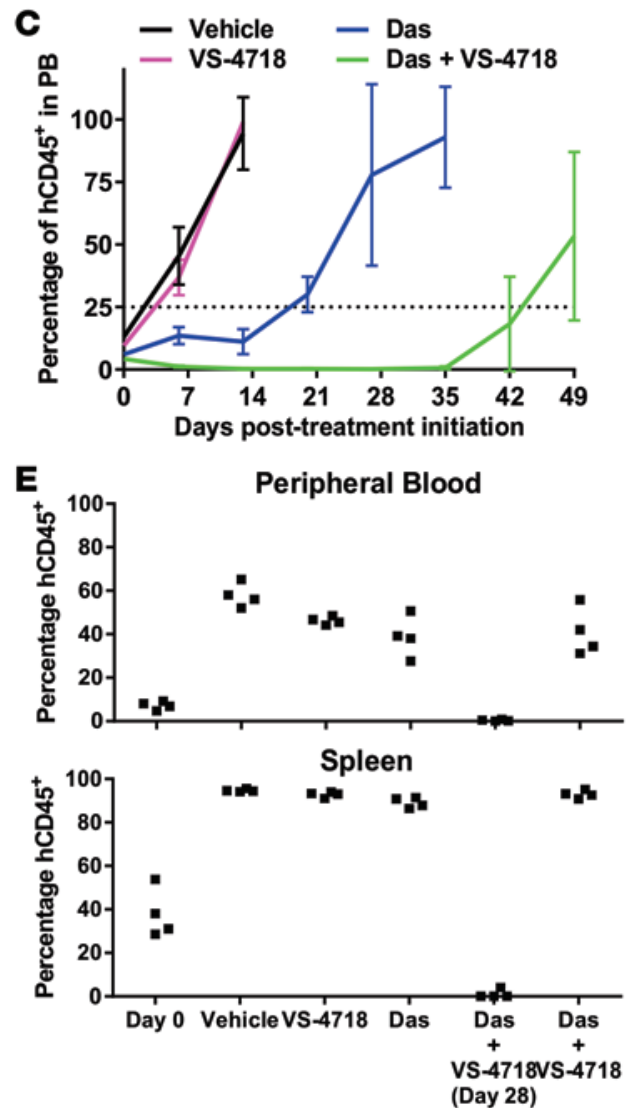

$\mathbf{F}$

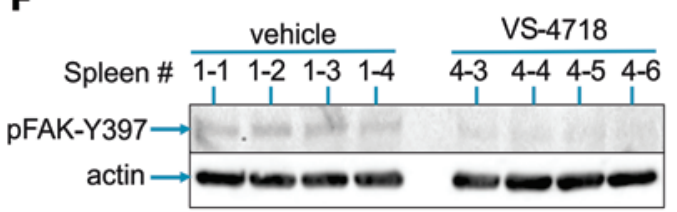

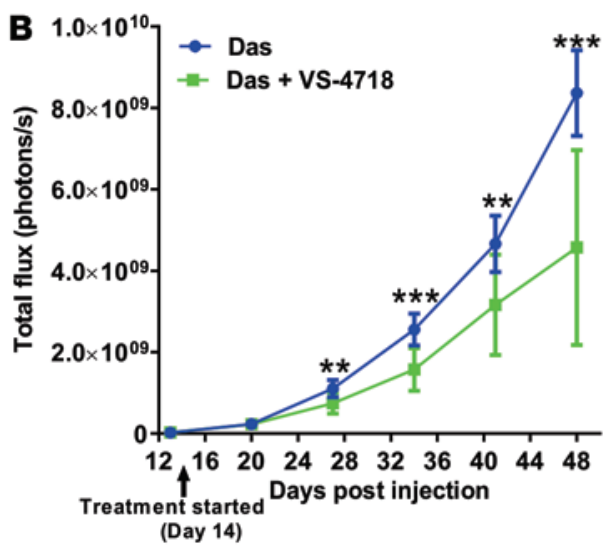

D

- Vehicle - Das

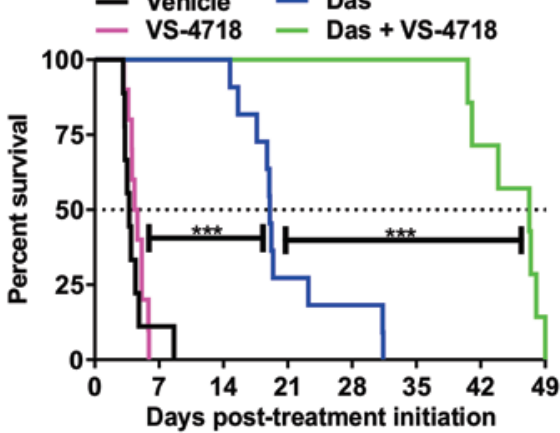

Cardiac Puncture
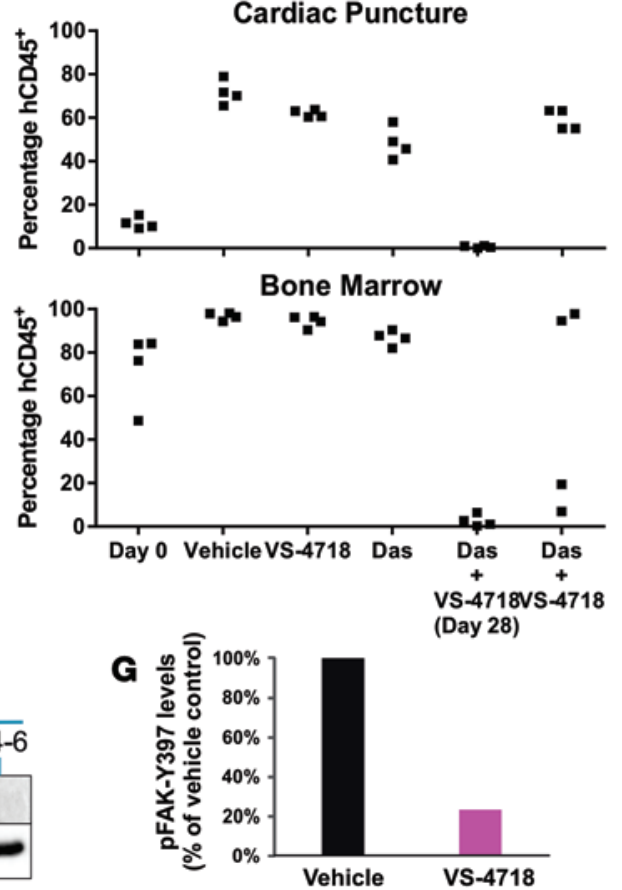

Figure 6. Addition of VS-4718 to dasatinib therapy regimen decreases the leukemic burden of xenografted human BCR-ABL1 B progenitor acute lymphoblastic leukemia in vivo and improves survival. (A) Live whole-animal quantification of in vivo luciferase activity to monitor the progression of luciferase-tagged h9407 B progenitor acute lymphoblastic leukemia cells during treatment with vehicle compared with VS-4718 as a single agent or (B) dasatinib in combination with VS-4718 versus dasatinib as a single agent. (C) The percentage of human CD45 (hCD45') ALL-4 cells in peripheral blood (PB) was determined by flow cytometric analysis to track progression of leukemic cells while on treatment. (D) KaplanMeier survival curve of mice in $\mathbf{C}$. (E) Enumeration of hCD45+ ALL-4 cells from PB, cardiac puncture, spleen, and bone marrow at the time of sacrifice of mice in $\mathbf{C}$ and D. A sentinel cohort of dasatiniband VS-4718-treated mice were sacrificed at the end of treatment on day 28 to confirm remission.

(F) Western blot analysis of spleen samples from mice on in vivo ALL-4 xenograft treatment, showing inhibition of phosphorylation of FAK at residue Y397 (pFAK-Y397). (G) Quantification of pFAK-Y397 levels by ELISA with OD levels normalized to total protein. Data represent averages $\pm \mathrm{SD}$ in $\mathbf{A}-\mathbf{C} ; n=9-11$ mice in $\mathbf{A}-\mathbf{D}$ and $n=4$ mice in $\mathbf{E}-\mathbf{G}$; ${ }^{* *} P \leq$ 0.005 , ${ }^{* *} P \leq 0.0005$, Mantel-Cox test. Das, dasatinib.

was potently effective in killing leukemic cells and reversing adhesion in vitro and in vivo. A significant, although less striking, effect was observed in $I K Z F 1$ wild-type, $\mathrm{Ph}^{+} \mathrm{ALL}$, supporting the notion that $\mathrm{BCR}-$ ABL1 may directly activate FAK signaling and that FAK inhibition may be efficacious in all cases of ALL, regardless of $I K Z F 1$ status.

These results provide a compelling rationale for clinical evaluation of combined ABL and FAK inhibition in $\mathrm{Ph}^{+}$ALL. Single-agent ABL1 inhibition has transformed the outcome of $\mathrm{Ph}^{+} \mathrm{CML}$, resulting in deep and prolonged remissions in the majority of patients (23). However, $\mathrm{Ph}^{+} \mathrm{CML}$ is a distinct disease from $\mathrm{ALL}$, in which $\mathrm{Ph}$ alone is not only required but is sufficient to induce a myeloproliferative neoplasm, 
and additional recurring genetic lesions are observed at transition to blast crisis, including IKZF1 at progression to $\operatorname{ALL}(2,24) . \mathrm{Ph}^{+} \mathrm{ALL}$ exhibits only transient responses to single-agent TKI therapy, and disease progression is frequent with chemotherapy alone (25-27). The combination of TKI with chemotherapy has improved outcomes (28), yet these remain inferior to non-IKZF1-mutated ALL $(29,30)$. Recent preclinical trials using the same $A r f^{\prime-}$ BCR-ABL1 pre-B model of B-ALL used in these studies suggest that pairing dasatinib with the Janus kinase inhibitor ruxolitinib and chemotherapy (dexamethasone) is a promising therapeutic approach in $\mathrm{Ph}^{+} \mathrm{B}-\mathrm{ALL}$, although the 2-drug combination of dasatinib and ruxolitinib was only modestly more effective than dasatinib alone (31). These experimental findings and clinical observations strongly suggest that directly disrupting the perturbations induced by IKZF1 alterations, either by restoring expression with retinoids or by disrupting the effects of integrin and FAK signaling, represent a powerful approach to improve outcome in this disease.

\section{Methods}

Expression vectors and retroviral production. cDNAs encoding BCR-ABL1 were cloned into mouse stem cell virus-internal ribosome entry site-GFP (MSCV-IRES-GFP [MIG]) (32) or luciferase (MSCV-IRES-luc2) vectors (33). cDNAs encoding wild-type dominant-negative IK6 were PCR amplified from human leukemic cell RNA (2) and cloned into MIG. Vectors were packaged into replication-incompetent ecotropic retroviral particles by transient transfection of 293T cells with a triple plasmid (pMD old gag pol and pCAG4-Eco) system.

Gene transduction and transplantation. MSCV vectors expressing human BCR-ABL1 and luciferase were used for transduction of whole bone marrow from $\mathrm{Arf}^{\prime-}$ or $I \mathrm{kzf1} \mathrm{I}^{+/-}(34,35)$ mice and generation of in vitro pre-B cell cultures $(32,36)$. Upon establishment of BCR-ABL1-transformed cultures after 7 to 8 days, cells were transduced with MSCV retroviral supernatants expressing IK6 and GFP or empty vector (MIG). Cells were GFP sorted prior to subsequent culture and/or transplantation into mice. All pre-B cell cultures were maintained in RPMI supplemented with $10 \%$ fetal calf serum, penicillin, streptomycin, glutamine, and $55 \mu \mathrm{M} \beta$-mercaptoethanol (BCM10). For all pre-B cell transplantation experiments, 200,000 BCRABL1-expressing pre-B cells were transplanted by tail vein injection into sublethally (5 Gy) irradiated 8- to 10 -week-old C57BL/6 recipients. Animals were monitored daily and sacrificed when moribund or upon clinically manifest central nervous system involvement.

Generation of xenografted mice. A human $\mathrm{Ph}^{+}$IK6 B-ALL sample (h9407) was provided by John Dick from the University Health Network, Toronto, Ontario, Canada (37); ALL-4 samples were generated as previously reported (38). h9407 cells were transduced for 3 hours with pCL20-MSCV-Luciferase-ires-YFP viral supernatant, washed 3 times in PBS, and transplanted by tail vein injection into unirradiated immunodeficient NSG mice. After recipients were moribund with disease, $\mathrm{YFP}^{+}$cells were sorted and used for in vivo treatment studies. Disease progression was monitored either by flow cytometric analysis of peripheral blood cells stained for hCD45 or whole body xenogen imaging following injection of D-luciferin to detect luciferase activity.

In vivo drug treatment studies. Dasatinib (LC Laboratories) was administered by oral gavage at $10 \mathrm{mg} / \mathrm{kg}$ (C57BL/6), $2 \mathrm{mg} / \mathrm{kg}$ (NSG h9407), or $15 \mathrm{mg} / \mathrm{kg}$ (NOD/SCID ALL-4) in $80 \mathrm{mM}$ citric acid (pH 3.1). VS-4718 in $0.5 \%$ carboxymethyl cellulose (Sigma-Aldrich) and $0.1 \%$ Tween 80 (Sigma-Aldrich) in sterile water was administered by oral gavage at $50 \mathrm{mg} / \mathrm{kg}$. Mice were treated once daily with dasatinib (C57BL/6 and NSG h9407) or 5 days per week (NSG ALL-4).

Calvarial imaging. Calvaria were harvested in triplicate at 72 hours after transplant, placed in PBS, and immediately imaged imaged using a 40× 1.1 NA LD C-Apochromat water immersion objective on a Zeiss LSM 780 microscope (Carl Zeiss Microscopy). Internal detectors were used, and the GFP signal was spectrally unmixed from the td-Tomato signal. Excitation was with a Coherent Chameleon Ti:Sapphire laser tuned to $900 \mathrm{~nm}$. Three $200 \times 200$ micron fields of view within bone marrow cavities were analyzed per calvarium. Bone marrow cavities were identified by the absence of a second harmonic signal from the surrounding bone. For enumeration of round versus spindle-like cells, the investigator was blinded to the treatment group of calvarial images.

$R N A$ and protein sequencing. Leukemic cell mRNA and protein sequencing was performed as previously described (4). In brief, RNA was extracted using TRIzol reagent and quantitated by fluorimetry (Qubit), and integrity was assessed using a TapeStation 2200 (Agilent Technologies). RNA sequencing was performed using Truseq poly-A selected library construction (Illumina) and paired end 100-bp sequencing 
using Hiseq 2000 and 2500 sequencers. Proteins were extracted from pre-B cells and digested in solution by sequential addition of Lys-C and trypsin proteases. The resulting peptides were individually desalted and labeled with 6-plex Tandem Mass Tag reagents (Thermo Scientific) for sequencing by tandem mass tag-based mass spectrometry.

Reverse-phase protein array analysis. Freshly harvested patient-derived xenografts, h9407 (IK6) and TB-08-3679 (IKZF1 wild type), were harvested and treated with $0.1 \mu \mathrm{M}$ VS-4718 or DMSO for 24 hours at $37^{\circ} \mathrm{C}, 8 \% \mathrm{CO}_{2}$. Cell lysates were prepared according to the specifications of Theranostic Health, which performed the arrays.

In vitro apoptosis, viability, and colony-formation assays. To analyze the effects of VS-4718 on apoptosis, one million cells were seeded in triplicate wells of a 6-well dish and treated with 0,1 , or $3 \mu \mathrm{M}$ of drug for 24 hours prior to propidium iodide staining to determine DNA content and annexin V-APC staining to assess cell viability. Cells were analyzed on a BD FACSCalibur (BD Biosciences). For cell viability assays, murine pre-B cells or human xenograft cells from freshly harvested bone marrow were plated in 96-well plates and treated with VS-4718 and dasatinib dissolved in DMSO for 48 hours at the indicated concentrations. Cells were incubated for 4 hours with resazurin and read on a Synergy HT (Biotek). For assays testing the effects of VS-4718 on colony formation, quadruplicate 96 -well plates containing $0.1 \mu \mathrm{M}$ of drug in $200 \mu 1$ media/ well were seeded with 1 pre-B cell per well for each of the indicated genotypes via a BD FACSAria II cell sorter (BD Biosciences). Plates were incubated at $37^{\circ} \mathrm{C}, 8 \% \mathrm{CO}_{2}$, for 7 days before colonies were counted. To test the effects of drugs on colony formation, drug was added to the media in each plate prior to sorting.

Adhesion assays. Arf ${ }^{\prime-}$ BCR-ABL1 empty vector (MIG) and $A r f^{\prime-}$ BCR-ABL1 IK6-IRES-GFP pre-B cells were pretreated with continuous rotation for 3 hours with $1 \mu \mathrm{M}$ VS-4718 and plated in triplicate at 6 $\times 10^{6}$ cells per well in nontissue culture treated 6-well plates coated with RetroNectin (Takara Bio). Plates were centrifuge at $450 \mathrm{~g}$ for 5 seconds, and cells were allowed to adhere for 30 minutes, followed by mild agitation of the plate and aspiration of unbound cells in the supernatant. For combination assays, dasatinib was added at $10 \mathrm{nM}$ for the 30-minute incubation. Wells were lightly washed with PBS prior to dissociation and collection of adherent cells for counting on a Bio-Rad TC10 automated cell counter.

Immunoblotting and ELISA. Spleen fragments from control or VS-4718-treated mice (50 mg/kg) were frozen in RNALater and lysed in mPER buffer (ThermoFisher Scientific) with $3 \times$ protease/phosphatase inhibitors (Halt Protease and Phosphatase Inhibitor Cocktail). For lysates from RetroNectin assays, pre-B cells adhering to RetroNectin monolayers were lysed by addition of mPER buffer directly to the well. Western blotting was performed using anti- p-P130Cas and anti-pFAK-Y397 antibodies (Cell Signaling Technologies, 4015 and 3283, respectively). ELISA was done using the FAK [pY397] Phospho-ELISA Kit (ThermoFisher Scientific, KHO0441).

Accession numbers. RNA-sequencing data have been deposited in the Gene Expression Omnibus (accession number GSE68391).

Statistics. All graphs and Kaplan-Meier survival curves were generated and analyzed using GraphPad Prism Version 6.0 (GraphPad). All data are presented as mean \pm SD. Significance was determined using 2-tailed Student's $t$ test, 2-way ANOVA, or Mantel-Cox log-rank test as appropriate. A $P$ value of less than 0.05 was considered significant.

Study approval. Mice were housed in an American Association of Laboratory Animal Care-accredited facility and were treated using Institutional Animal Care and Use Committee-approved protocols in accordance with NIH guidelines.

\section{Author contributions}

MLC, IMS, JAP, DTW, RBL, PJH, MAS, and CGM designed experiments. MLC, KE, JR, AR, LJ, and IMS performed experiments. MLC and IMS analyzed data. MLC and CGM wrote the manuscript.

\section{Acknowledgments}

We thank the St. Jude Children's Research Hospital Flow Cytometry and Cell Sorting Shared Resource and Small Animal Imaging Facility. Images were acquired at the Cell and Tissue Imaging Center, which is supported by St. Jude Children's Research Hospital and National Cancer Institute grant P30 CA021765-35. This work was partially supported by the American Lebanese Syrian Associated Charities of St. Jude Children's Research Hospital and grants from the National Cancer Institute (NOI-CM-42216 and NOI-CM-91001-03). C.G. Mullighan is a St. Baldrick's Foundation Scholar. R.B. Lock is supported by a fellowship from the 
National Health and Medical Research Council. Children's Cancer Institute Australia is affiliated with University of New South Wales Australia and the Sydney Children's Hospitals Network.

Address correspondence to: Charles G. Mullighan, St. Jude Children's Research Hospital, 262 Danny Thomas Place, Mail Stop 342, Pathology, Memphis, Tennessee 38105, USA. Phone: 901.595.3387; E-mail: charles.mullighan@stjude.org.

1. Mullighan CG, et al. Genome-wide analysis of genetic alterations in acute lymphoblastic leukaemia. Nature. 2007;446(7137):758-764.

2. Mullighan CG, et al. BCR-ABL1 lymphoblastic leukaemia is characterized by the deletion of Ikaros. Nature. 2008;453(7191):110-114.

3. van der Veer A, et al. IKZF1 status as a prognostic feature in BCR-ABL1-positive childhood ALL. Blood. 2014;123(11):1691-1698.

4. Churchman ML, et al. Efficacy of retinoids in IKZF1-mutated BCR-ABL1 acute lymphoblastic leukemia. Cancer Cell. $2015 ; 28(3): 343-356$

5. Joshi I, et al. Loss of Ikaros DNA-binding function confers integrin-dependent survival on pre-B cells and progression to acute lymphoblastic leukemia. Nat Immunol. 2014;15(3):294-304.

6. Richardson A, Parsons JT. A mechanism for regulation of the adhesion-associated protein tyrosine kinase pp125FAK. Nature. 1996;380(6574):538-540.

7. Sanders MA, Basson MD. Collagen IV-dependent ERK activation in human Caco-2 intestinal epithelial cells requires focal adhesion kinase. J Biol Chem. 2000;275(48):38040-38047.

8. Schlaepfer DD, Hunter T. Evidence for in vivo phosphorylation of the Grb2 SH2-domain binding site on focal adhesion kinase by Src-family protein-tyrosine kinases. Mol Cell Biol. 1996;16(10):5623-5633.

9. Mitra SK, Hanson DA, Schlaepfer DD. Focal adhesion kinase: in command and control of cell motility. Nat Rev Mol Cell Biol. 2005;6(1):56-68.

10. Westhoff MA, Serrels B, Fincham VJ, Frame MC, Carragher NO. SRC-mediated phosphorylation of focal adhesion kinase couples actin and adhesion dynamics to survival signaling. Mol Cell Biol. 2004;24(18):8113-8133.

11. Sulzmaier FJ, Jean C, Schlaepfer DD. FAK in cancer: mechanistic findings and clinical applications. Nat Rev Cancer. 2014;14(9):598-610

12. Roberts KG, et al. Genetic alterations activating kinase and cytokine receptor signaling in high-risk acute lymphoblastic leukemia. Cancer Cell. 2012;22(2):153-166.

13. Mullighan CG. Molecular genetics of B-precursor acute lymphoblastic leukemia. J Clin Invest. 2012;122(10):3407-3415.

14. Virely C, et al. Haploinsufficiency of the IKZF1 (IKAROS) tumor suppressor gene cooperates with BCR-ABL in a transgenic model of acute lymphoblastic leukemia. Leukemia. 2010;24(6):1200-1204.

15. Heltemes-Harris LM, et al. Ebf1 or Pax5 haploinsufficiency synergizes with STAT5 activation to initiate acute lymphoblastic leukemia. J Exp Med. 2011;208(6):1135-1149.

16. Liu GJ, et al. Pax5 loss imposes a reversible differentiation block in B-progenitor acute lymphoblastic leukemia. Genes Dev. 2014;28(12):1337-1350.

17. Zhang J, et al. Harnessing of the nucleosome-remodeling-deacetylase complex controls lymphocyte development and prevents leukemogenesis. Nat Immunol. 2012;13(1):86-94.

18. Dege C, Hagman J. Mi-2/NuRD chromatin remodeling complexes regulate B and T-lymphocyte development and function. Immunol Rev. 2014;261(1):126-140.

19. Bottardi S, et al. The IKAROS interaction with a complex including chromatin remodeling and transcription elongation activities is required for hematopoiesis. PLoS Genet. 2014;10(12):e1004827.

20. Oravecz A, et al. Ikaros mediates gene silencing in T cells through Polycomb repressive complex 2. Nat Commun. 2015;6:8823.

21. Nishii K, et al. Non-DNA-binding Ikaros isoform gene expressed in adult B-precursor acute lymphoblastic leukemia. Leukemia. 2002;16(7):1285-1292.

22. Tankoni I, et al. PND-1186 FAK inhibitor selectively promotes tumor cell apoptosis in three-dimensional environments. Cancer Biol Ther. 2010;9(10):764-777.

23. Yeung DT, et al. TIDEL-II: first-line use of imatinib in CML with early switch to nilotinib for failure to achieve time-dependent molecular targets. Blood. 2015;125(6):915-923.

24. Iacobucci I, et al. Identification and molecular characterization of recurrent genomic deletions on 7p12 in the IKZF1 gene in a large cohort of BCR-ABL1-positive acute lymphoblastic leukemia patients: on behalf of Gruppo Italiano Malattie Ematologiche dell'Adulto Acute Leukemia Working Party (GIMEMA AL WP). Blood. 2009;114(10):2159-2167.

25. Bernt KM, Hunger SP. Current concepts in pediatric Philadelphia chromosome-positive acute lymphoblastic leukemia. Front Oncol. 2014;4:54.

26. Arico M, et al. Clinical outcome of children with newly diagnosed Philadelphia chromosome-positive acute lymphoblastic leukemia treated between 1995 and 2005. J Clin Oncol. 2010;28(31):4755-4761.

27. Fielding AK. How I treat Philadelphia chromosome-positive acute lymphoblastic leukemia. Blood. 2010;116(18):3409-3417.

28. Schultz KR, et al. Long-term follow-up of imatinib in pediatric Philadelphia chromosome-positive acute lymphoblastic leukemia: Children's Oncology Group study AALL0031. Leukemia. 2014;28(7):1467-1471.

29. Biondi A, et al. Imatinib after induction for treatment of children and adolescents with Philadelphia-chromosome-positive acute lymphoblastic leukaemia (EsPhALL): a randomised, open-label, intergroup study. Lancet Oncol. 2012;13(9):936-945.

30. Cortes JE, et al. A phase 2 trial of ponatinib in Philadelphia chromosome-positive leukemias. $N$ Engl J Med. 2013;369(19):1783-1796. 
31. Appelmann I, et al. Janus kinase inhibition by ruxolitinib extends dasatinib- and dexamethasone-induced remissions in a mouse model of $\mathrm{Ph}^{+}$ALL. Blood. 2015;125(9):1444-1451.

32. Williams RT, Roussel MF, Sherr CJ. Arf gene loss enhances oncogenicity and limits imatinib response in mouse models of BcrAbl-induced acute lymphoblastic leukemia. Proc Natl Acad Sci U S A. 2006;103(17):6688-6693.

33. Boulos N, et al. Chemotherapeutic agents circumvent emergence of dasatinib-resistant BCR-ABL kinase mutations in a precise mouse model of Philadelphia chromosome-positive acute lymphoblastic leukemia. Blood. 2011;117(13):3585-3595.

34. Kamijo T, et al. Tumor suppression at the mouse INK4a locus mediated by the alternative reading frame product p19ARF. Cell. 1997;91(5):649-659.

35. Wang JH, Nichogiannopoulou A, Wu L, Sun L, Sharpe AH, Bigby M, Georgopoulos K. Selective defects in the development of the fetal and adult lymphoid system in mice with an Ikaros null mutation. Immunity. 1996;5(6):537-49.

36. Whitlock CA, Witte ON. Long-term culture of murine bone marrow precursors of B lymphocytes. Methods Enzymol. 1987;150:275-286.

37. Notta F, et al. Evolution of human BCR-ABL1 lymphoblastic leukaemia-initiating cells. Nature. 2011;469(7330):362-367.

38. Lock RB, et al. The nonobese diabetic/severe combined immunodeficient (NOD/SCID) mouse model of childhood acute lymphoblastic leukemia reveals intrinsic differences in biologic characteristics at diagnosis and relapse. Blood. 2002;99(11):4100-4108 